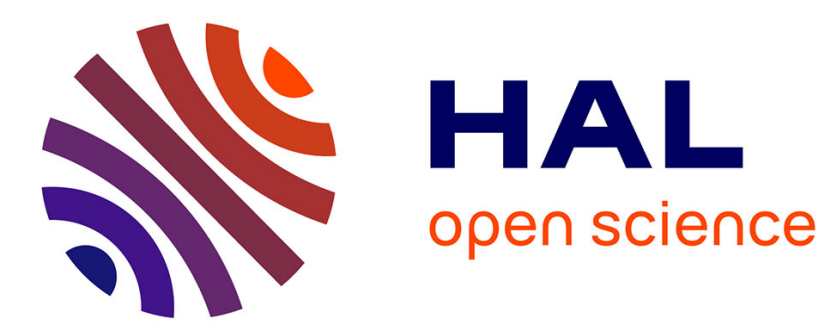

\title{
Multiagent Simulation of Real-Time Passenger Information on Transit Networks
}

Mahdi Zargayouna, Amine Othman, Gérard Scemama, Besma Zeddini

\section{To cite this version:}

Mahdi Zargayouna, Amine Othman, Gérard Scemama, Besma Zeddini. Multiagent Simulation of Real-Time Passenger Information on Transit Networks. IEEE Intelligent Transportation Systems Magazine, 2020, 12 (2), pp.50-63. 10.1109/MITS.2018.2879166 . hal-02877206

\section{HAL Id: hal-02877206 https://hal.science/hal-02877206}

Submitted on 14 Sep 2020

HAL is a multi-disciplinary open access archive for the deposit and dissemination of scientific research documents, whether they are published or not. The documents may come from teaching and research institutions in France or abroad, or from public or private research centers.
L'archive ouverte pluridisciplinaire HAL, est destinée au dépôt et à la diffusion de documents scientifiques de niveau recherche, publiés ou non, émanant des établissements d'enseignement et de recherche français ou étrangers, des laboratoires publics ou privés. 


\title{
Multiagent Simulation of Real-Time Passenger Information on Transit Networks
}

\author{
Mahdi Zargayouna, Amine Othman, and Gérard Scemama \\ Université Paris Est, IFSTTAR, COSYS, GRETTIA, 14-20, \\ boulevard Newton, 77447 Champs sur Marne, France. \\ E-mail: \{hamza-mahdi.zargayouna, amine.othman, gerard.scemama\}@ifsttar.fr
}

Besma Zeddini

Quartz, EISTI, Avenue du Parc, 95000 Cergy Pontoise, France.

E-mail: besma.zeddini@eisti.eu

\begin{abstract}
Modeling and simulation play an important role in transportation networks analysis. With the widespread use of personalized real-time information sources, the status of the simulation depends heavily on individual travelers reactions to the received information. As a consequence, it is relevant for the simulation model to be individual-centered, and agent-based simulation is the most promising paradigm in this context. Information is now personalized, and the simulations have to take into account the interaction of individually guided passengers. In this paper, we present a multiagent simulation model to observe and assess the effects of real-time information provision on the passengers in transit networks. These effects are measured by simulating several scenarios according to the ratio of connected passengers to a real-time information system. We represent the passengers and the vehicles as agents in the system. We analyze the simulated scenarios following their effect on the passengers travel times. The information provided to the connected passengers is based on a space-time representation of the transportation networks. Results show that real-time personalized information may have an increasingly positive impact on overall travel times following the increasing ratio of connected passengers. However, there is a ratio threshold after which the effect of real-time information becomes less positive.
\end{abstract}

\section{Introduction}

o define mobility policies, the decision makers need support systems to assist them. In this context, simulation is one of the important tools that allow them to test strategies and multiple scenarios without impacting the real traffic [1], [17], [25], [39]. However, transportation systems are becoming

Digital Object Identifier 10.1109/MITS.2018.2879166 Date of publication: $x x x x x x$ progressively complex as they are increasingly composed of smart and mobile entities. Indeed, passengers mobile devices and connected vehicles allow passengers and vehicles to have up-to-date information and their behavior is now related to this information. It is now possible to provide passengers with optimal itineraries and also to update these itineraries in real-time, following the dynamic status of the networks (congestions, breakdowns, accidents, etc.). Providing passengers with traffic information has 
generally a positive effect on them and improves traffic by alleviating congestion and reducing waiting and travel times. However, the broadcast of traffic information and the individual guidance of passengers might have adverse effects and impact negatively the traffic status.

These possible side effects have been listed in [28]. The authors state that three phenomena could be observed as a side effect of the use of advanced traveler information systems. First, when passengers receive too much information, they tend to ignore this information and try to find an itinerary on their own (oversaturation). Second, if the same itineraries are provided to passengers who have the same transportation preferences, congestion might be created (concentration). Finally, if the same alternative is provided to too many passengers, the original congestion could be simply moved to another location (overreaction).

In this paper, we focus on public transportation networks and we evaluate the advantages and the limits of the provision to passengers of real-time traffic, notably in disturbed conditions. In this context, simulation is one of the important tools that allow strategies testing and multiple scenarios [1], [17]. Simulations can be used to validate the impact of the use of cooperative systems [19], to test changes in traffic after the introduction of new mobility services, such as carpooling, dial a ride, etc.

Our proposal is based on the multiagent paradigm, which is relevant paradigm for the design and implementation of transportation applications. In [4], the authors state multiple reasons to use multiagent systems in transportation applications. Among other arguments, the authors indicate that the solving of several transportation problems with multiagent systems is natural and intuitive. The simulation of passengers mobility for instance is particularly suitable for an agent-based design. Indeed, the objective in this kind of simulations is to take into account human behaviors, interacting in an open, dynamic and complex environment [6]. In this application, passengers perceive individual information, and make individual decisions, while being situated in and interacting with an environment (the transportation network and the information sources) on which they have partial and incomplete information. This configuration obeys the general definitions of agents as entities that: i) are situated in some environment, ii) that are capable of autonomous action on it [36], iii) that can perceive this environment and iv) that have a partial and incomplete perception of it [14].

In this paper, we propose an agent-based traffic simulator for transit networks. Built on top of the Repast Simphony platform, it allows to represent vehicles and travelers movements on the networks. It also represents information flows between agents and between the operator and the agents. The simulator allows us to simulate and evaluate the effects of real-time traveler information on transit networks.

The primary objective of this work is to verify the effect of the use of real-time information, including personalized information, on the transportation network status. Our results validate the fact that this impact is positive to a certain extent. However, it also points out that, starting from a certain ratio of equipped travelers with smartphones, personalized information is no longer beneficial.

This paper is structured as follows. In section II, we present the previous proposals for the evaluation of the effects of information on passengers and we discuss the choice of the simulation platform. In section III, we detail the simulation platform that we propose. We present the data and parameters of the simulation. We also describe the agent behaviors and the itineraries calculation. In section IV, we describe the management of information flows and disturbances in the simulation, using a space-time representation of the environment. In section $\mathrm{V}$, we describe the configuration of our experimental study and we provide the results. We finally conclude and describe some further work we are conducting.

\section{State of the Art}

\section{A. Impact of Passenger Information}

The effects of information on passengers travel and transportation networks have been generally investigated in the literature using either surveys or simulations. Works that use surveys investigate the passenger's experience feedback (e.g. [8], [12] and [30]) and generally concern small scales (in terms of number of passengers and network size). This is due to the difficulty and the high cost of studies with a big number of passengers. The findings of these studies are more reliable because they are based on actual observations, but they are not easily generalizable because they are limited in time and space and concern a small number of observations. For these reasons, a large number of works have chosen simulation as an evaluation tool. Simulation is able to extend the experimentation field and makes it possible to test a big number of scenarios. Scenarios can integrate different levels of information and different network conditions. In the following, we present the simulation proposals for evaluating the effects of passenger information on transit networks.

In [11], the authors simulate the passenger's decision process after the reception of real-time information. This process is in charge of the computation and the choice of the passenger's itinerary and its execution. In [9], the passenger decision model is divided into two sub-models: the generator of the set of choices and the itinerary choice model. In [16], the authors also use a dynamic itinerary choice model to manage the passenger decision process. In [15], the authors propose a passengers information model that manages the information provision to passengers in the simulation model. In this approach, the personalized information resulting from the use of smartphones is not directly considered. The equipped passengers are 
represented as entities with a high access level to realtime information (e.g. in [9] and [13]). Different levels of information are generally used in the experimentations as in [13] where 6 access levels to real-time information are studied. Results reported in [11] and in [9] show that providing more comprehensive real-time information may lead to path choice shifts and time savings. They recommend to provide real-time information at the station level to enable more informed decisions and gains in travel time.

Individual real-time information on smartphones is not explicitly considered in the literature. Indeed, the realtime information evaluated in the studied works concern only localized information (screen displays and voice messages in the stops and stations). This kind of information differs from personalized information, accessible via smartphones, that assist passengers during their movements by providing specific information about their itinerary. This kind of information represents an increasingly important element in the advanced travelers information systems and plays a significant role in modern transportation systems. In the work presented in this paper, we consider the equipped passengers as a distinct category, with a specific behavior. We analyze the results according to the two types of passengers (equipped and non-equipped).

Results reported in the literature also concern small networks or only some couples of origins-destinations. The results in the work presented in this paper concern the data of a big network with thousands of edges. We also consider thousands of passengers representing up to $25 \%$ of the real population of the considered network.

\section{B. Choice of the Simulation Platform}

Since we desire to model individual and heterogeneous behaviors of passengers, we choose the multiagent paradigm for the system modeling. Several multiagent simulators for passenger mobility exist in the literature. MATSim [23] is a platform for micro-simulation of traffic that is widely known and used. AgentPolis [18] is an agent-based platform for multimodal traffic. Miro [10] simulates the urban dynamics of a population and proposes a multiagent simulation to test planning scenarios, while Transims [26] represents travelers movements in multimodal networks and assesses the effects of the policy changes in traffic. However, none of these proposals considers connected passengers and the impact of their level of information on the network. In the proposal of this paper, passengers that are connected to real-time information sources have their routes monitored continuously. Alternatives are also proposed to them if they encounter disturbances during their trip.

As a result of this study on existing multiagent simulators for passengers mobility, we decide to design and implement a new simulator. To design and implement a multiagent simulator, there are mainly two possibilities. It is either possible to directly use a programming language, or to use a multiagent simulation platform. We choose to use an existing agent-based platform because the implementation is faster and more efficient. Two criteria have guided our platform choice. First, we desire to be able to deploy the simulations on many servers, which is one of our ongoing works. The second capacity that we are looking for is the ability of the simulation platform to create geospatial models, i.e. its ability to handle geographic information. Based on these criteria, we have studied multiple agent-based platforms such as Jade [5], Mason [22], Gama [31] and Repast Simphony [32]. We believe that Repast Simphony is the platform that meets most our criteria. The platform integrates a GIS library (Geotools), and provides advanced geographic services (graph modeling, shortest paths, visualization of 2D and 3D data, etc.). It also offers an easy integration of distribution platforms such as Terracotta and Gridgain. This is why the simulator is based on this platform.

\section{Multiagent Transit Simulator}

Our proposal is an agent-based platform that simulates passengers mobility. Differently from state of the art approaches, the information flows in the network and personalized information are considered. Fig. 1 presents the multiagent system (MAS), the agents that compose it, the environment and the interaction between agents. The passenger agents and the vehicle agents are the agents that move on the network. There are two kind of passenger agents: connected passenger agents (to a real-time information source) and non-connected passenger agents. The local-information agents are responsible for sharing disturbances information in the network stops. The simulation platform is responsible for the planning and the monitoring of the connected passengers trips based on a spatiotemporal network (presented in section IV).

The simulator represents itinerary planners, passengers and their movements, public transportation vehicles and their movements, and information devices. To do so, input data and some additional parameters are needed. In Fig. 2, we provide the successive steps followed by a simulation. A simulation first loads the parameters (the duration of a simulation, the number of agents in the simulation, etc.). The graphs representing the transportation networks are then created (described in section III-A) and the scheduler is launched. The scheduler is a central component in the simulation since it synchronizes the execution of the agents. It schedules the execution of the agents for each tick of simulated time. The actions executed in one simulated tick of time are considered as being simultaneous. At each tick of simulation, the agents are executed in parallel threads ${ }^{1}$ (to take advantage of multicore architectures), while the scheduler waits for them to finish the execution of the actions planned for the current tick, before incrementing the

${ }^{1}$ In our experiments, we use 12 cores. 


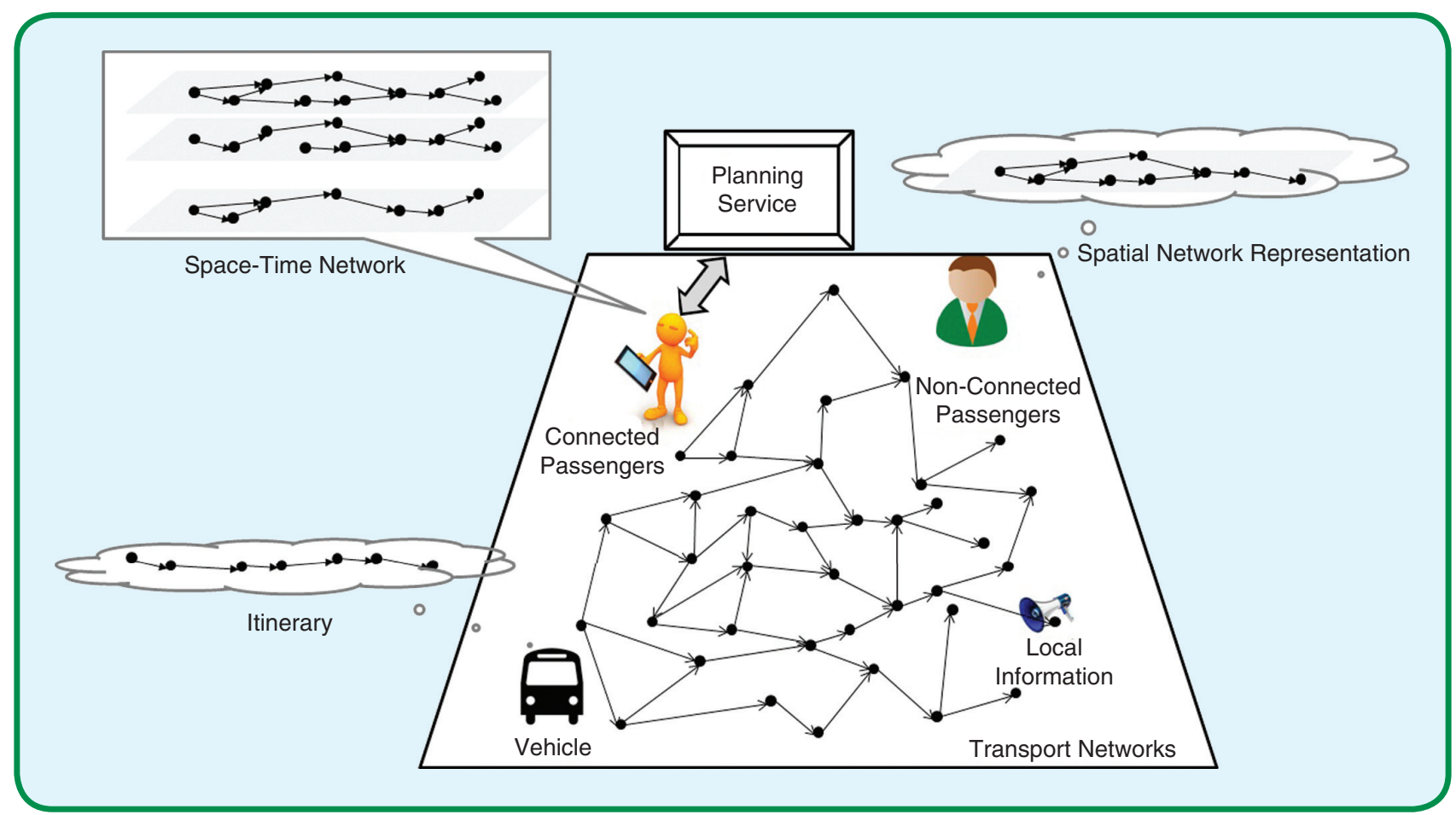

FIG 1 Multiagent system model.

tick and starting over again. When launched, each agent executes a step method, in which he executes the behaviors described later (compute an itinerary, move on the network, get available information, wait for a vehicle, etc.). Hence there is a main program with a scheduler that controls the simulation, launches the agents and synchronizes their actions, while between each two time ticks, agents are executed in parallel. When the simulation ends, we collect the results and end the simulation run. The collected results concern all the travelers itineraries and some indicators, such as the travel times and waiting times for the travelers.

\section{A. Multiagent System}

The multiagent system is composed of three types of agents (connected passenger agents, non-connected passenger agents and vehicle agents) and a planning service.

\section{1) Planning Service}

The planning service has the responsibility of calculating the itinerary of the connected passenger agents. The service always considers the newest known status of the networks (described in section III-A 5). An itinerary is made of consecutive edges (either in the pedestrian network or the public transportation network) together with their corresponding visit times.

\section{2) Vehicle Agents}

The vehicle agents represent public collective transportation vehicles that have a defined itinerary and a timetable.
Vehicles infer their paths from the timetable input data. Each vehicle agent moves at each simulation time tick with the allowed distance following his current speed. At each visited stop, the onboard passengers who have a transfer at this stop leave the vehicle and the passengers waiting for this vehicle at the stop take him. While moving, the vehicle agent moves his onboard passengers to the same coordinates at the same time. At each simulation tick, the vehicle agent checks if he has reached his destination (the last stop in his itinerary). If so, he is removed from the simulation.

\section{3) Passenger Agents}

Passenger agents represent the users of the transit network. There are two types of passenger agents: connected and non-connected to a real-time information system using smartphones. If the passenger is connected, he receives an itinerary from the planning service. If he is not connected, he uses a spatial mental representation of the network to compute his path (cf. section IV-D). To travel, the passenger agent loops over three states: walking, waiting for a vehicle and being on board of a vehicle, until he reaches his destination. When he is on board of a vehicle, a passenger agent delegates the control of his movements to the corresponding vehicle agent.

\section{4) Agent Movements}

Each passenger and each vehicle agent knows the coordinates that he has to sequentially move to, following his computed path. Depending on the type of agent and his 


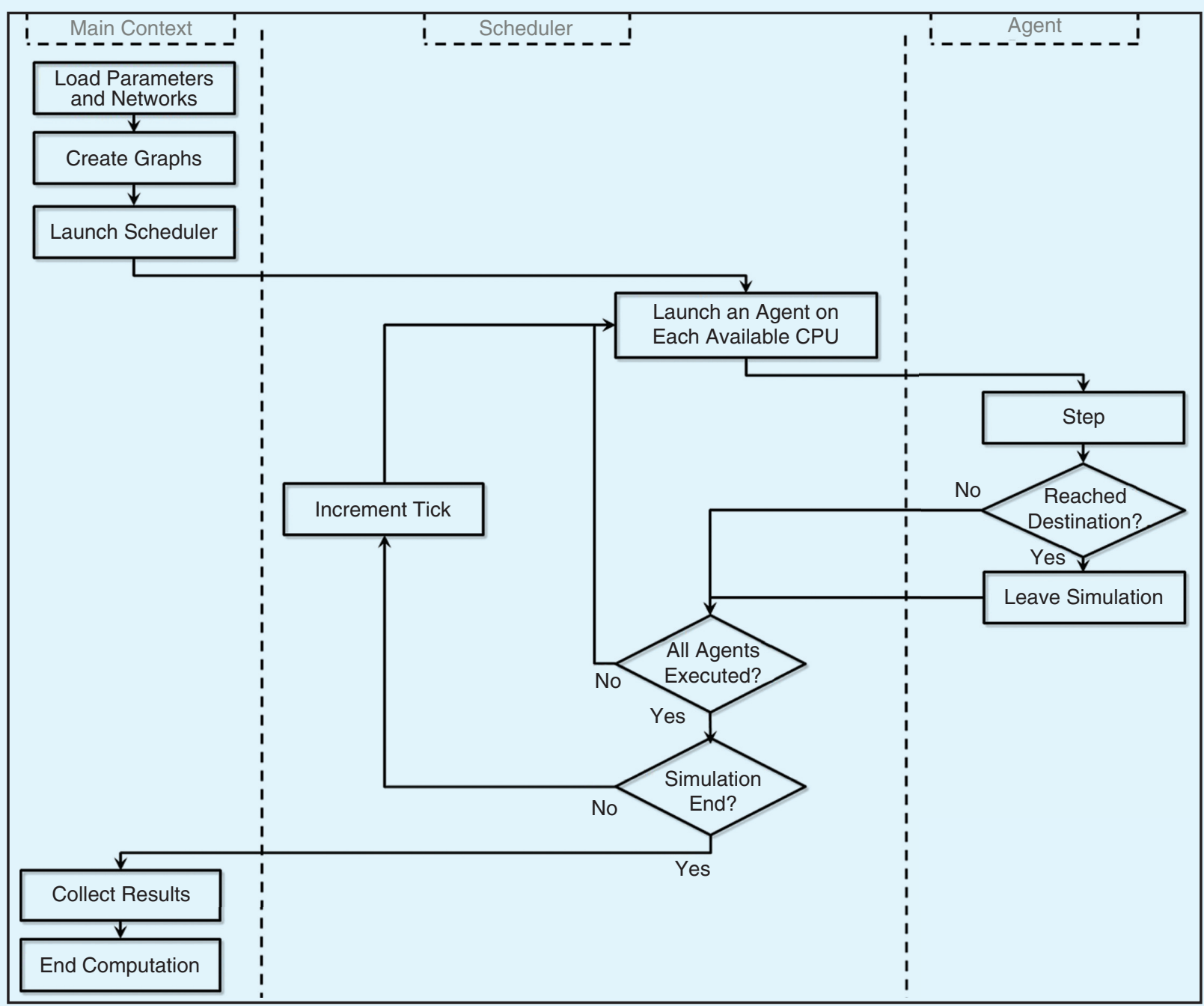

FIG 2 Steps of a simulation.

corresponding speed, he can move for only a certain distance at each simulated tick of time.

\section{5) Itinerary Planning}

The itinerary planning of passengers is composed of two elements. The first element concerns the walking parts of the itinerary, while the second concerns the public transportation parts of the itinerary. Starting from an origin position, the passenger agent searches for the closest stops contained in a geographic circle around him². The same procedure is applied for the destination node. The result is two sets $S_{o}$ (for origin stops) and $S_{d}$ (for destination stops) of public transportation nodes as possible origins and destinations, respectively. All the shortest paths between

${ }^{2}$ The radius of the circle is inferred from passengers profiles (the accepted walking distance) when they exist. It is otherwise set to a default value, 500 meters for instance. $\left(s_{o}, s_{d}\right)$ pairs $\in S_{o} \times S_{d}$ are computed, and the $\left(s_{o}, s_{d}\right)$ pair corresponding to the shortest path (including the walking path) is chosen. The calculation of the public transportation part of the path is based on a frequency-based shortest path method [3].

The public transportation network is composed of nodes (the stops) and edges (the links between stops). The costs associated with the edges are defined according to the frequencies on these edges. The travel time $t_{i j}^{k}$ between two nodes $i$ and $j$ of the same itinerary $k$ is equal to the current travel time on the edge $t_{i j}$ plus the average waiting time in seconds, equal to $3600 / \phi(k)$, with $\phi(k)$ the hourly frequency of the itinerary $k$.

To avoid having passengers shortest paths with many transfers, we need to differentiate the nodes following their itineraries (recall that a transportation line is composed 
of a set of itineraries). We modify the public transportation network as follows (cf. Fig. 3). Let $\left\langle s_{1}, s_{2}\right\rangle$ be an edge in the original public transportation graph. Let $i t_{1}$ and $i t_{2}$ be two itineraries using this edge. We create four new vertices: $i t_{1}: s_{1}$ and $i t_{1}: s_{2}$ that are connected with the edge that belongs to $i t_{1}$; and $i t_{2}: s_{1}$ and $i t_{2}: s_{2}$ that are connected with the edge that belongs to $i t_{2}$. We also create four new edges $\left\langle i t_{1}: s_{1}, i t_{2}: s_{1}\right\rangle,\left\langle i t_{2}: s_{1}, i t_{1}: s_{1}\right\rangle,\left\langle i t_{1}: s_{2}, i t_{2}: s_{2}\right\rangle$ et $\left\langle i t_{2}: s_{2}, i t_{1}: s_{2}\right\rangle$, that we call transfer edges and which cost is equal to the average pedestrian travel time between the two stops (if any). With this graph, shortest paths will encourage passengers to stay on the same vehicle, and a transfer is proposed when it is either impossible or too costly to stay on the same vehicle or the same line. Finally, the $A^{*}$ shortest path algorithm is applied on the resulting network.

After applying the shortest path algorithm, the planning service interrogates the stops of the best found itinerary to infer the sequence of vehicles that the passenger agent will have to take and sends back the result to the passenger agent.

\section{Passenger Information Integration}

In this section, we describe the integration of passenger information in the simulation.

\section{A. Space-Time Representation of the Environment}

The notification about disturbances in public transportation networks and the replanning of routes of passengers is a complex task. Indeed, the changes in the itineraries and travel times are exogenous to the agents and these latter do not know a priori where and when they may occur.

On the one hand, we have the real-time information providers that produce dynamic information. In the other hand, we have passenger agents who are potentially interested in this information. The broadcast of dynamic information to all passengers agents is a simple and intuitive method for the matching of passengers and information providers, but this method is expensive because it generates unnecessary processing and uses unnecessary bandwidth. It would also directly lead to the oversaturation adverse effect described in the introduction.

Several approaches exist in the literature for matching agents who don't know each other a priori (e.g. middleagents [35] and recommendation systems [34]). We adopt an environment-centered approach, which focuses on the shared data and allows for the selection of relevant information by the agents without having to know or to maintain knowledge about the emitters of these data. Our environment-based approach is based on a representation model of the transportation environment in the form of a space-time network. This representation has been used in the past in different applications: dial a ride, vehicle routing, etc. [37], [38]. In our application, the multiagent space-time environment represents the state of the public

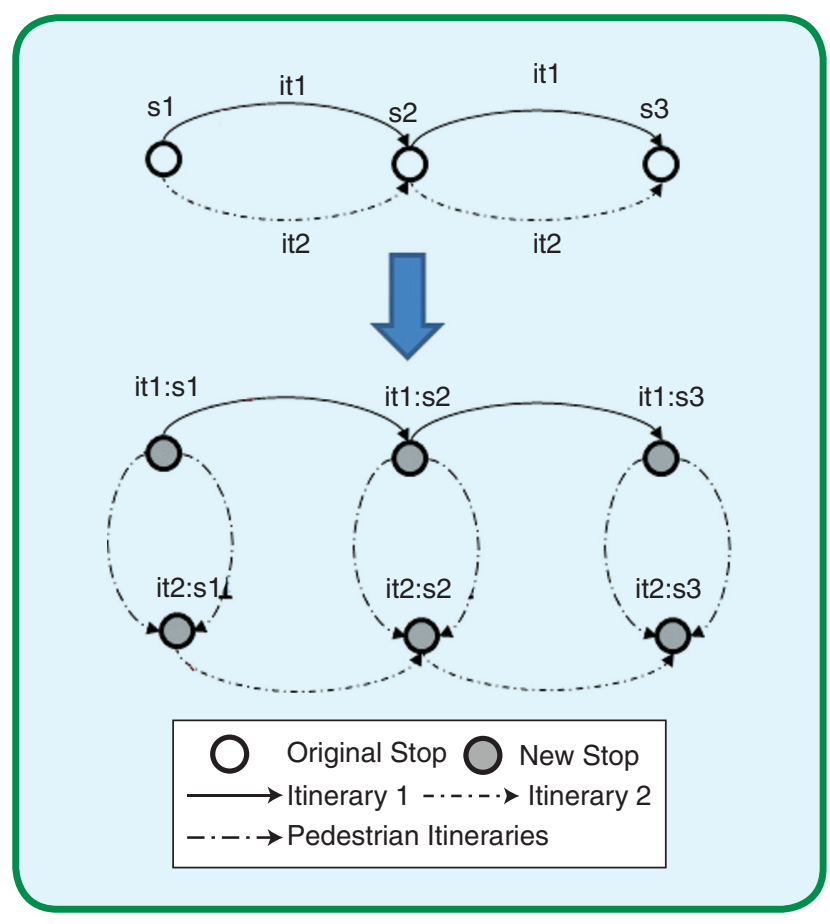

FIG 3 Network Transformation.

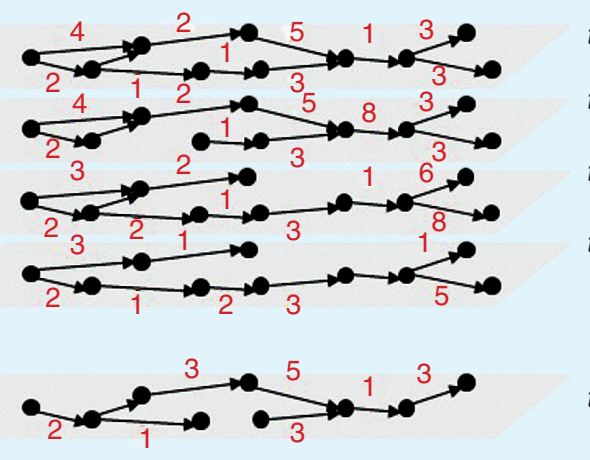

$t_{0}$

$t_{1}$

$t_{2}$

$t_{3}$

$t_{H}$

FIG 4 Spatiotemporel graph.

transportation network through time. This environment is the main interlocutor for connected passenger agents and is active, in the sense that it stores information and triggers reaction when relevant events occur.

Consider the transportation network $G=(V, E)$, with a node set $V=\left\{\left(v_{i}\right)\right\}, i=\{0, \ldots, N\}$ and an edge set $E=\left\{\left(v_{i}, v_{j}\right) \mid v_{i} \in V, v_{j} \in V, v_{i} \neq v_{j}\right\}$. Let $D$ and $T$ be two matrices of costs, $D=\left\{\left(d_{i j}\right)\right\}$ and $T=\left\{\left(t_{i j}\right)\right\}$, of dimensions $N \times N$ (the link $\left(v_{i}, v_{j}\right)$ has $d_{i j}$ as distance and $t_{i j}$ as travel time). The space-time representation of the multiagent environment is made of $H$ copies of $G$, where $H$ depends on the considered time frame for the application ${ }^{3}$ :

${ }^{3}$ For instance, if we consider a timeframe of two hours, and a discretized time of one minute, $H=120$. 
Table I. Implementation of the space-time graph in the simulator.

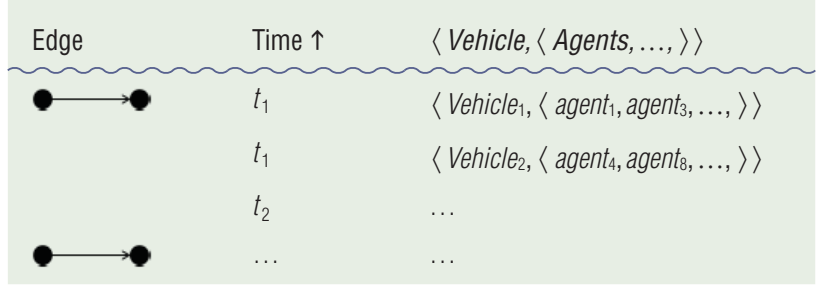

$G(t)=(V(t), E(t))$, with $V(t)$ the set of nodes at time $t$ and $E(t)$ the set of directed links at time $t$ with $0 \leq t \leq H$ (cf. Fig. 4). The cost matrix $T$ becomes $T(t)$ to be timerelated as well. At each progression of time, the $t_{0}$ version of the space-time network is deleted, $t_{1}$ becomes $t_{0}$ and $t_{H}$ is created.

This structure is generic and may have a different semantics depending on the considered application. The temporal copies of $G$ are generally not identical. Indeed, for road networks, we can have different travel times between two copies to reflect the traffic state. In crisis management, edges and nodes may be present in a copy and absent in another copy to reflect the expansion of a crisis. Edges can be absent to reflect vehicles's timetables and disturbance situations, as in the application of this paper.

An agent who wish to be informed by only changes occurring on a node $v$ of the network during a period ranging from $t_{1}$ to $t_{2}$ have to register with nodes $\left\{\left(v, t_{1}\right), \ldots,\left(v, t_{2}\right)\right\}$. As we show in the following, with this representation of the environment, every dynamic modification of the transportation offer is directly reported to the only concerned agents, which avoids massive dissemination of information to all the system agents.

In the following, we describe the use of this structure to inject disturbances and disseminate general and personalized information to both connected and non-connected passengers.

In the simulator, we have implemented the space-time network in the form of a map (cf. Table. I). The keys to the map are the edges of the spatial graph. The corresponding values are sorted lists of times related to start times of vehicles from the origin node of the edge. To each time value we associate a pair $\langle$ vehicle, $\langle$ agents $\rangle\rangle$, corresponding to the concerned vehicle and all the agents who have subscribed to the corresponding space-time edge. When a vehicle's timetable changes, the concerned edges are identified. Then the map is requested with these edges as keys, and the $\langle$ vehicle, $\langle$ agents, .. $\rangle\rangle$ pairs corresponding to the old visit times are retrieved from the map. Finally, all the agents in the lists are notified about the changes. The agents that may subscribe to space-time edges are local information agents and connected passenger agents, as described in the following.

\section{B. Local Information Agents}

Local information agents represent devices that broadcast traffic information on screens or through voice announcements at the stops. The information broadcasted by the local information agents concern events generally occurring elsewhere in the network. To get this information, local information agents subscribe to the space-time edges of the public transportation lines that they are interested in. In our experiments, the local information agents subscribe to the lines that pass by their stop. Indeed, we make the realistic choice to broadcast on stops the only information that concerns the lines passing by them. Otherwise, the amount of information in the stops would be too difficult to display on the screens or to enounce through voice messages. When dealing with different networks, the choice of the subscription strategy can be adapted in consequence. Only passenger agents present in a stop can perceive the locally broadcasted information.

\section{Connected Passengers Behavior}

Passengers that are connected to a real-time information source receive their calculated path from the planning service. When they receive the computed path, connected passengers interact with the space-time network. Indeed, to be aware of the only traffic events that concern him, a passenger agent subscribes to the edges of the space-time that form his itinerary (that he received from the planning service). When the path of the passenger is impacted by a disturbance, the information is received from the spacetime network. The planning process is executed again, and the planning service will base his calculation on the new configuration and the new status of the network.

\section{Non-Connected Passengers Behavior}

Passengers that are not connected to a real-time information source ground their path calculation on a personal view of the network. They follow the calculated path and wait at the programmed stops. They deviate from their path in two cases. Either they are actually caught in a disturbance; or they are informed about a disturbance, via announcements in the stop. If they receive such an alert, they integrate the modifications in their mental view of the transportation network, and calculate a new path using that view.

\section{E. Injecting Disturbances}

To verify the effects of real-time information on passengers, both with personalized information and with local information, we need to model disturbances. Indeed, as indicated in the state of the art, information is most important in case of disturbances, when passengers need to find new routes and need to be oriented on the network. Disturbances are reflected by modifying the space-time network. Indeed, to inject delays in the model, we dynamically modify visit times of the vehicles while to model breakdowns 
and disconnections, we delete all the concerned spacetime edges. When the timetable of a vehicle is modified, all the concerned agents are notified of the change in their plan. These agents are unsubscribed of these space-time edges, and the planning service calculates new itineraries for them, with new subscriptions.

\section{F. Temporal Model of the Simulation}

The concurrent execution of agents, especially with a parallel scheduler, necessitates the synchronization of their access to the transportation environment, to avoid incoherent states of the network due to simultaneous modification. Thus, the calls to the movement methods on the network are synchronized: passenger and vehicle agents, once they take their movement decision, move sequentially (while in the same time tick). Other considerations concerning the execution order of the agents have also to be taken into account. Indeed, the main interactions between agents are performed between:

- passenger agents and vehicle agents at the stops;

- passenger agents and local information agents at the stops;

- connected passenger agents and the planning service;

- connected passenger agents and the space-time networks.

The order in which agents are activated by the scheduler might alter the coherent outcome of these interactions. On the one side, since the vehicles arrive at the stops and look for passenger agents who plan to take him, there is a risk that a passenger agent who wants to take the vehicle is not yet at the stop, not because he is late, but because the scheduler has not activated him yet for the current simulation time tick. For this reason, passenger agents are activated by the scheduler first, before the vehicle agents. On the other side, incoherences in passengers reactions to disturbances might also occur. For instance, we could have passengers who react immediately to disturbances, while others only react at the next time tick, because the disturbance injection is performed in the middle of agents execution for a simulation time tick. To avoid this situation, disturbances and their impact on the space-time network and on the local information agents are performed in priority before activating passenger and vehicle agents.

\section{Experiments and Results}

\section{A. Setup}

The experiments are executed with the data of the city of Toulouse in France (cf. Fig. 5). We choose this French city because we have detailed data about its network and a description of the travel patterns of the region [27]. The data come from Tisséo-SMTC, the public transportation authority of the Greater Toulouse. The public transportation network of Toulouse is composed of 80 lines,

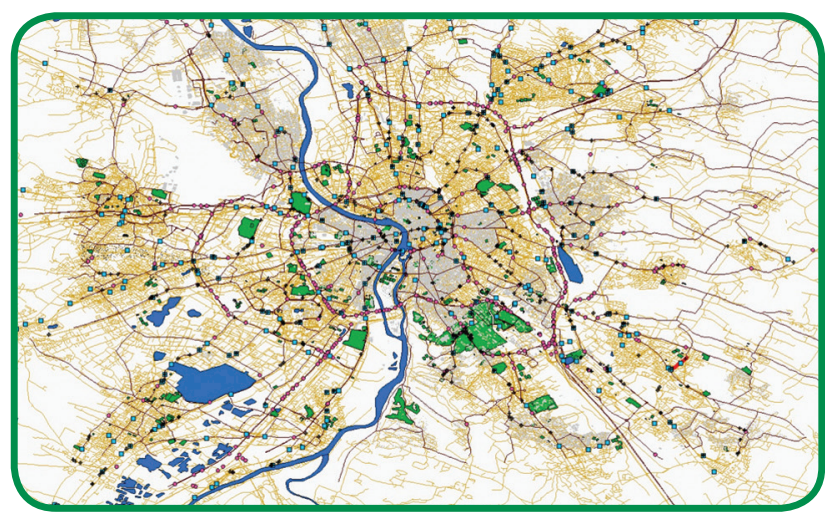

FIG 5 Screenshot of a simulation execution (Toulouse city).

359 itineraries and 3,887 edges. Frequencies and edges costs are updated hourly. The multiagent system is made of 18,180 vehicles and from 5,000 to 30,000 passengers. We define the number of ticks per simulation to 5,000 for a journey from 6 am to 2 am. Every simulated tick corresponds to approximately 14 seconds. The origins and destinations of passengers are randomly chosen in a way that is coherent with travel patterns of the region (the originsdestinations generation method is described in [21]). We have executed the simulations on a PC under Windows 7 with a processor Intel Xeon CPU E5-2630 (12 cores at $2 \mathrm{Ghz}$ ) with $50 \mathrm{~GB}$ of memory.

Previous works have shown that traveler information has little impact in case of small disturbances. For this reason, we decide to use serious disturbances instead in the form of complete disconnection of edges. In every simulation, we have generated 5 random edge disconnections on the network during the whole simulation (one disconnection every 233 real time minutes approximately). Every disconnection lasts 250 simulated ticks (slightly less than one hour in real time). Due to edge disconnections, some passengers cannot find an itinerary to their destination anymore, because edges disconnection impacts network connectivity. In the following results, these passengers are not considered ${ }^{4}$. Disturbances are random but concern only a certain number of edges which we have considered as significant to disconnect: the edges through which pass at least 5 different itineraries. The 5 randomly disconnected edges are chosen between 21 candidate edges that satisfy this requirement.

\section{B. Scenarios}

We consider six different information level scenarios (cf. Table II) and each one is executed 25 times. The first scenario is "the reference configuration" (to which we compare all the others) where no up-to-date information are provided to the passengers, neither local nor personalized. They only have

${ }^{4}$ The ratio of passengers without itinerary is stable, around $5 \%$ in all the simulations. 
Table II. Information level in each scenario.

$\begin{array}{lll}\text { No } & \text { No } \\ 1 & \text { Yes } & \text { No } \\ 2 & \text { Yes } & 20 \% \\ 3 & \text { Yes } & 50 \% \\ 4 & \text { Yes } & 80 \% \\ 5 & \text { Yes } & 100 \%\end{array}$

Table III. The bus capacities in the simulator.

\begin{tabular}{|c|c|}
\hline Passengers Number & Vehicles Capacity \\
\hline 1,000 & 1 \\
\hline 5,000 & 4 \\
\hline 10,000 & 8 \\
\hline 20,000 & 17 \\
\hline 30,000 & 25 \\
\hline
\end{tabular}

$10,000,20,000$ and 30,000$)$ can be seen as an increasing precision for the same simulation. That means that, with 1,000 simulated travelers for instance, each traveler agent represents around 100 human travelers, with 5,000 simulated travelers, each traveler agent represents 20 human travelers, and so on ${ }^{5}$. We report the average results and the observed standard deviations.

In case of disturbances, vehicle capacity limitation is one of the main causes of passengers delays. Thus, vehicle capacities have to be chosen carefully to avoid simulation bias. Indeed, if vehicle capacity is too big, the effect of disturbances might be underestimated. On the contrary, if vehicles capacity is too small, it can artificially amplify the impact of disturbances by introducing a big waiting time in the stops. Since we simulate a fraction of the real number of passengers, we adapt the bus capacity proportionally to this fraction. We base our calculation on the 2010 annual data of the bus network of Tisseo [33]. Our goal is to have an equivalent ratio between the daily passengers number, the number of available places in the vehicles (seating and standing) and the daily number of passengers. The result of this adaptation is reported in the Table III.
Table IV. Synthesis of travel time improvement (Average (Standard deviation)).

$\begin{array}{llllll} & 1,000 & 5,000 & 10,000 & 20,000 & 30,000 \\ 0 \% & 0.45 \%(0.03 \%) & 1.45 \%(0.1 \%) & 7.56 \%(0.25 \%) & 5.43 \%(0.52 \%) & 3.44 \%(0.33 \%) \\ 20 \% & 3.56 \%(0.23 \%) & 8.25 \%(0.57 \%) & 7.97 \%(0.26 \%) & 8.43 \%(0.8 \%) & 10.57 \%(1 \%) \\ 50 \% & 5.15 \%(0.33 \%) & 12.21 \%(0.84 \%) & 15.64 \%(0.52 \%) & 11.62 \%(1.1 \%) & 14.40 \%(1.36 \%) \\ 80 \% & 8.99 \%(0.58 \%) & 11.63 \%(0.8 \%) & 16.3 \%(0.54 \%) & 10.43 \%(0.99 \%) & 12.35 \%(1.17 \%) \\ 100 \% & 4.95 \%(0.32 \%) & 10.24 \%(0.71 \%) & 12.99 \%(0.43 \%) & 8.2 \%(0.79 \%) & 8.82 \%(0.84 \%)\end{array}$

the static description of the network and timetables. In the second scenario, only local information are given. The new travel times are available for the only passengers that are present in the considered stop. We do not consider any connected passenger in this scenario. In the remaining scenarios (3, 4, 5 and 6), local information are provided to passengers at the stops, and personal information is only available for the connected passengers. We consider $20 \%, 50 \%, 80 \%$ then $100 \%$ of connected passengers respectively in these scenarios. In the scenarios with local information (all the simulations except the reference configuration), we have placed local information agents in all the stops of the network. We report the average travel times for the passengers.

Every scenario is executed 25 times. Indeed, for each information level scenario, we consider increasing number of travelers: 1,000, 5,000, 10,000, 20,000 and 30,000 passengers, and every configuration (scenario and number of travelers) is executed 5 times to verify that the simulations are unbiased and results are reliable. The increasing number of simulated travelers $(1,000,5,000$,

\section{Results}

The results are reported in Table IV in which we provide the calculated average and the observed standard deviations $(\sigma)$. Results are reported in Fig. 6. The $\mathrm{x}$-axis represents the different equipment rates, from $0 \%$ to $100 \%$. The $\mathrm{z}$-axis represents the number of considered passengers $(1,000,5,000,10,000,20,000$ and 30,000). The y-axis represent the improvement rates. The curve represents the improvement rate in travel times in comparison with the reference configuration (with no information). It means that every point in the curve represents the improvement rate of the concerned simulation w.r.t the scenario with no information at all. Recall that the scenario with $0 \%$ connected passengers is the scenario where only local information is provided, which is different from the reference scenario in which there is no information at all. As we said earlier, we execute several simulations of every (equipment rate, number of passengers) pair to verify that the curve shape is not due to the origin-destination choices or from the difference in the injected disturbances, which are stochastic. Fig. 7 provides a sample result with 30,000 passengers, with a trend curve associated to the average values to facilitate the interpretation. As we see it in the table, the standard deviations are very

${ }^{5}$ The average number of travelers in the considered region is $100,000 \mathrm{hu}$ man travelers. 
low and do not question the trends of the curves.

Compared to the scenario without information, all scenarios improve the travel times. The lowest improvement $(0.45 \%)$ is related to the scenario with 1,000 passengers and $0 \%$ of connected passengers and the highest one $(16.3 \%)$ is related to the scenario with 10,000 passengers and $80 \%$ of connected passengers. It is clear that the reference scenario (without information) provides the worst average travel time, since all the improvement values are positive. Indeed, in this scenario passengers have the information about a disturbance when they are already blocked somewhere in the network. As a consequence, they look for alternative paths and use concurrently the rest of the transportation network. By doing so, they might

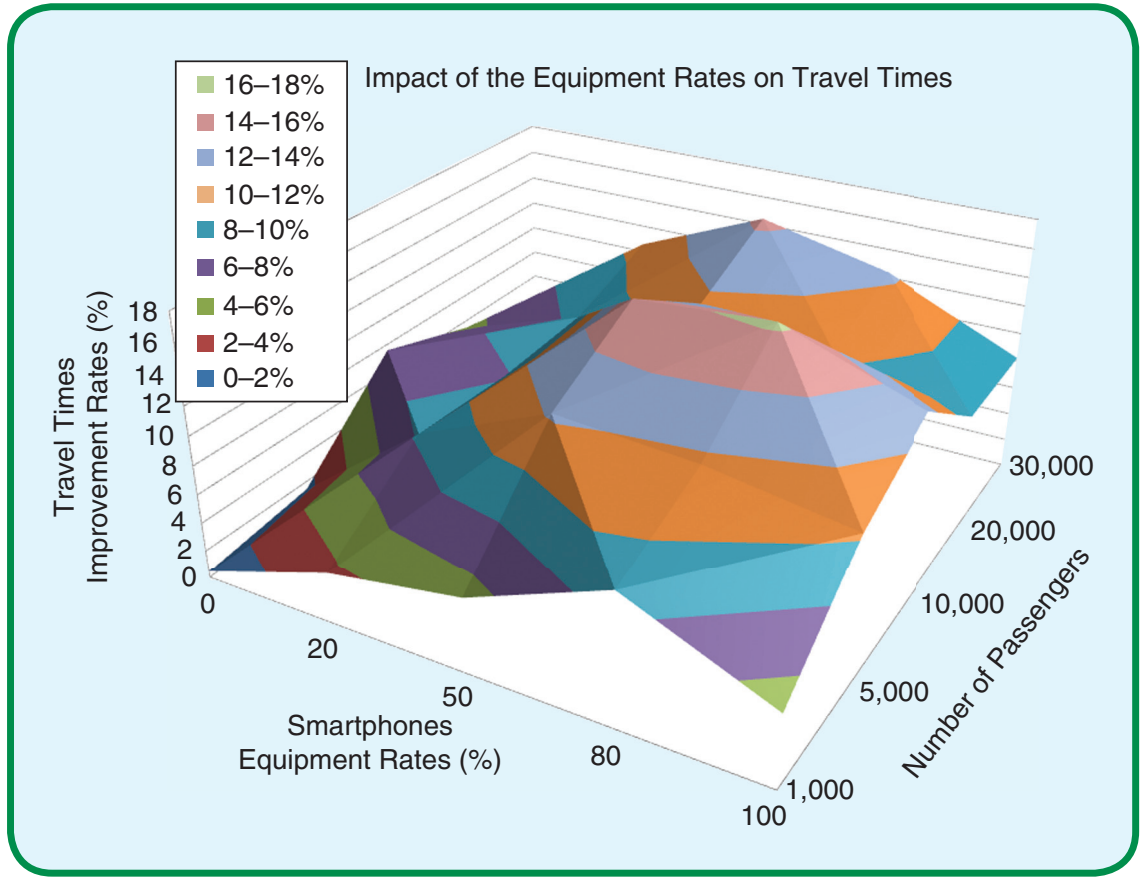

FIG 6 The improvements compared with the scenario without information (from 1,000 to 30,000 passengers). miss vehicles because of their capac-

ity constraints. They also could get stuck in another disturbance. The scenario 2, with local information and no personalized information, provides better results than the reference scenario. In this scenario, the passenger does not have necessarily to be present at the stops impacted by the disturbance to know that something is wrong. The passenger has the information when he visits a stop where local information is provided. However, this information is provided to him with a certain delay. Furthermore, since the path of the passenger is grounded on his partial vision of the network, he can head towards another disturbance.

The following scenarios, which integrate gradually more and more connected travelers, provide better results until $50 \%$ of connected passengers for scenarios with 5,000, 20,000 and 30,000 passengers and until 80\% for scenarios with 1,000 and 10,000 passengers. In these scenarios, the alternative paths are immediately computed and provided to the passengers. These paths are also based on the latest status of the network. As a consequence, the passengers avoid the disturbed zone early and do not risk to head towards another disturbance. The connected passenger does not have to be present at a stop where local information is provided, he receives a notification and a new plan immediately. However, this improvement is maximal at $50 \%$ of connected passengers for some scenarios and at $80 \%$ for others. After these thresholds, the improvement rate becomes lower with more connected passengers. In the scenarios where the majority of passengers is connected, in case of disturbances, the concerned passengers receive simultaneously new up-to-date plans. In this case, when they apply their new plans, they are faced with vehicles capacity constraints and will see their average travel times increase.

Starting from scenario 4 , the results start to become worse. Indeed, with $80 \%$ of connected passengers for scenario 5 and $100 \%$ of connected passengers for scenario 6 , most or all of the passengers get personal real-time information about disturbances in real-time and new up-todate plans are generated simultaneously. The consequence is that the passengers face capacity constraints of the vehicles and see their mean travel times increase. The results

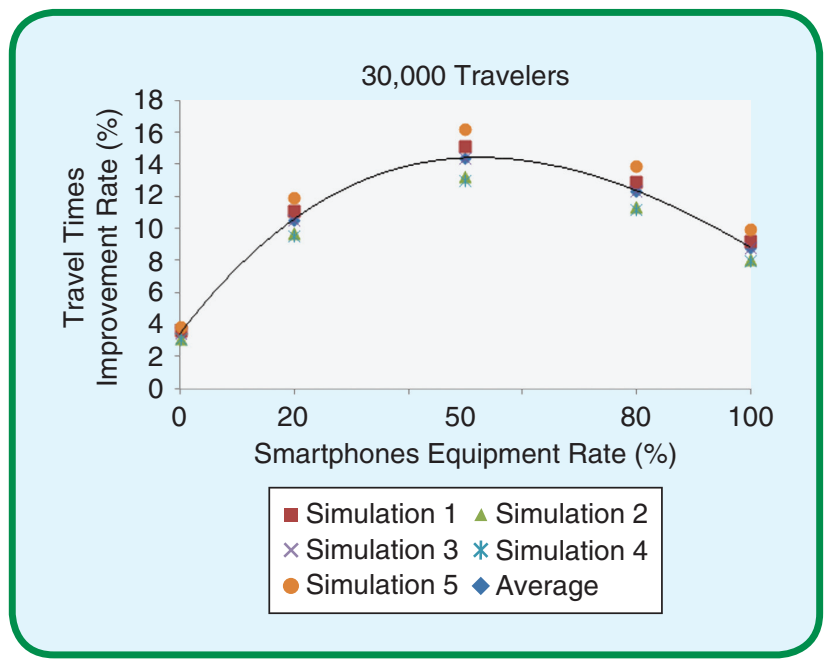

FIG 7 The improvements compared with the scenario without information (30,000 passengers). 


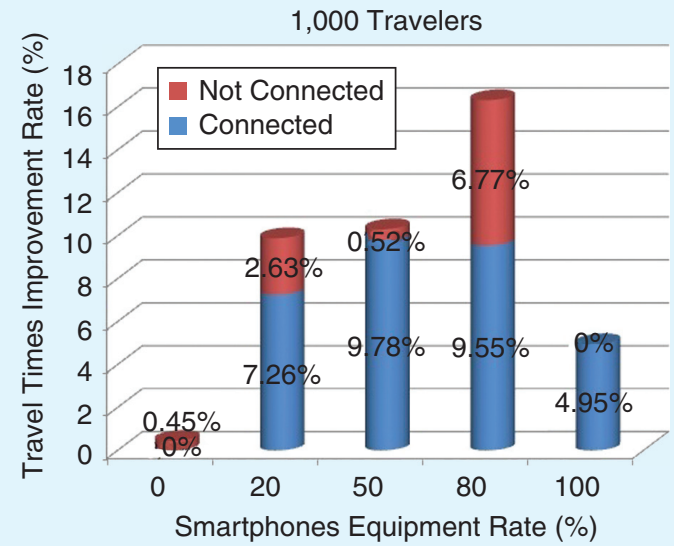

FIG 8 The compared improvements of travel times (connected passengers vs. non-connected passengers) with 1,000 passengers.

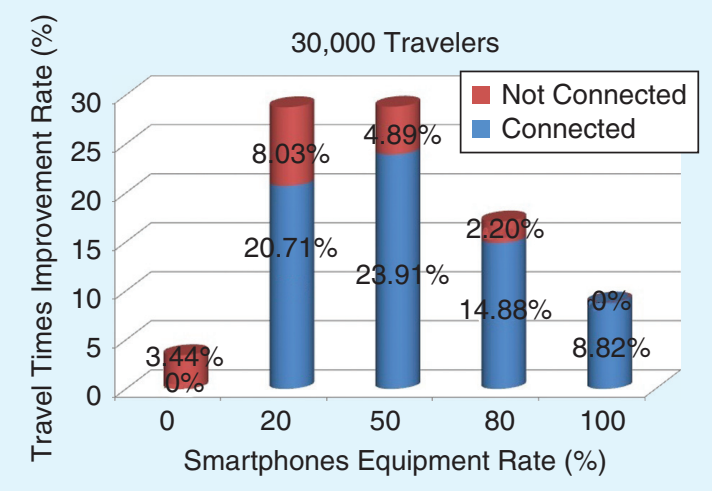

FIG 9 The compared improvements of travel times (connected passengers vs. non-connected passengers) with 30,000 passengers.

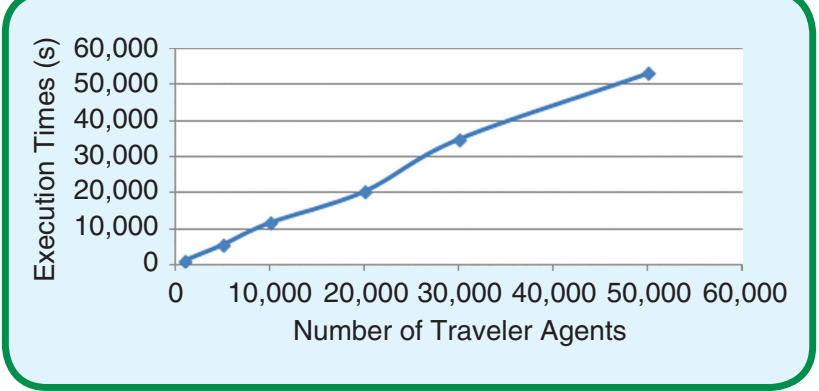

FIG 10 Average execution times.

are $15 \%$ worse for scenario 4 compared to scenario 3, and $23 \%$ worse for scenario 5 compared to scenario 4 .

Normally, the provision of real-time personalized information is supposed to have a positive impact, especially for connected passengers. To verify this impact on the only connected passengers, we provide the same results, but this time we differentiate improvement rates according to the passenger agent type (connected versus non-connected). The two example results are reported in the Figures 8 and 9 . We report in this figures the observed average values.

Every figure represents the improvements in travel times for a concerned number of passengers. The red part of the vertical bars of each figure represent the improvements for the non-connected passengers and the blue part represents the improvements for the connected passengers. Thus, for the scenarios with $0 \%$ of connected passengers, the improvement is ensured exclusively by the non-connected passengers and for the scenarios with $100 \%$ of connected passengers, the improvement is totally ensured by the connected passengers.

Results show that the improvement in travel times for the connected passengers follow the same trend that the curves in the figures 6 and 7, i.e. a growing improvement until a certain threshold before becoming lower.

However, this time, the maximum improvement is reached systematically at $50 \%$ of connected passengers. The improvement oversupply for the scenarios with 1,000 and 10,000 passengers and $80 \%$ of connected passengers is actually provided by the non-connected passengers.

The improvement rate for the non-connected passengers is quite stable between the scenarios with the same number of passengers. Thus, it is between $0.45 \%$ and $6.8 \%$ for 1,000 passengers, between $1.4 \%$ and $5.7 \%$ for 5,000 passengers, between $5.5 \%$ and $7.6 \%$ for 10,000 passengers, between $0.1 \%$ and $5.9 \%$ for 20,000 passengers, and between $2.2 \%$ and $8 \%$ for 30,000 passengers. Their curves don't follow a particular trend because their behavior is not directly impacted with the increase of the information level of other agents. Probably, the differences come from the (nondeterministic) processing order of the passenger agents when vehicles arrive at stops. If the number of passengers in a stop exceeds the vehicle capacity, no type of passengers is privileged to board. That is probably what gives this variations of the non-connected passengers' travel times.

\section{Computational Complexity}

The average execution times of our simulations are reported in Fig. 10. Computation times are correlated with the number of simulated travelers. Indeed, we have executed several simulations with vehicles only, and they were always very fast. Based on these results, we believe that we should further optimize our traveler agents, notably regarding the shortest paths computation.

In our experiments, we have chosen to execute several series of experiments, increasing the number of considered passengers. As we can see it, using bigger scales comes with a computational and resources cost. The different scales in our application provide the same conclusions: an increase of the benefits from personalized information, 
followed by a decrease. This fact suggests that there is no need, for this specific application, to use huge numbers of travelers.

\section{E. Discussion}

The most important result reported in this paper concerns the lower improvement in the passengers travel times starting from a certain ratio of connected passengers. This result may seem paradoxical, since it is intuitively expected that the average travel times continuously decrease when the ratio of connected passengers increases. In reality, this result is coherent with previous theoretical studies performed by [28] for road networks. Indeed, the phenomenon observed in this paper could be seen as a materialization of concentration and overreaction. Concentration is the consequence of a uniform perception of the network status by the passengers. The more connected passengers we have, the more uniform their perception will be. In our system, this uniform perception is stored in the space-time network, and indeed at $100 \%$ of equipment rate, all passengers have the exact same view of the network status. Overreaction is similar to concentration but is related to received traffic information, which make a fraction of the passengers transfer the congestion from one region to another. The more connected passengers in the system, the more substantial is the fraction that would follow the same paths and transfer the congestion. Congestion is mainly materialized by passengers who cannot take a vehicle because of capacity constraints. However, this relatively negative impact of information provision starts at very high threshold of connected passengers. Before that threshold, as it is the case in nowadays transportation systems, providing real-time personalized information does have an absolutely positive impact on the network status.

\section{Conclusion}

Surveys provide interesting results about the impact of passengers information on the status of transportation networks. Conducting such surveys on large scale is very costly, and simulation is an interesting tool to achieve such large-scale evaluation of the impact of passengers information. In this paper, we have provided the main components of a multiagent simulator for passenger mobility. This application allowed us to simulate information exchange and its effects on public transportation networks. The effects of two types of information (local information and personalized information) are measured by simulating different scenarios, and varying the ratio of connected passengers. Experiments have been executed on real scenario data from the city of Toulouse, France. The primary objective of this work was to verify the effect of the use of real-time information, including personalized information, on the transportation network status. Our results validate the intuition that this impact is positive to a certain extent. How- ever, we also found out that, starting from a certain ratio of equipped travelers with smartphones, personalized information is no longer beneficial.

In the near future, we plan to run experiments with hundreds of thousands of passengers to verify, among other objectives, that the results reported in this paper are still valid with real volumes of passengers, and without adaptation of vehicles capacities. In this context, the distribution of the simulation platform on the cloud [7], [29] is an ongoing work [24]. We are also expanding this work to multimodal networks and studying the use of powerful dynamic pathfinding algorithms like D*Lite [20]. Indeed, the frequency-based shortest path algorithm is fast and represents realistically the current path choices by the travelers, but is not necessarily optimal. In addition, we plan to integrate learning processes in the behavior of the traveler agents, which would also impact the itineraries calculation. The consideration of passengers preferences, other than the minimization of travel times (as in, e.g. [2]) is also interesting and is currently under investigation. Finally, the optimization of the passengers information strategies by the network operators is a main perspective of this work. Indeed, from a certain threshold of connected passengers, the operators can decide to optimize the information provided to passengers for a best management of their assignment on the network. Two interesting problems arise in this context. First, assigning travelers dynamically on different itineraries, taking into account realtime disturbances is an interesting optimization problem to tackle. Second, the compliance of passengers with the instructions, especially in this context (i.e. providing different itineraries for the same origin, destination and departure time) is interesting to verify. Again, surveys about this compliance level would be very useful.

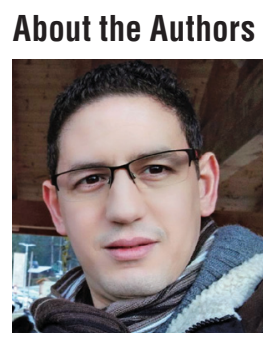

Mahdi Zargayouna received his M.Sc degree in computer science and the Ph.D. degree in computer science and artificial intelligence from the University of Paris Dauphine (France), in 2003 and 2007 respectively. Since 2008 , he is researcher in computer science and transportation science at IFSTTAR, the French Institute of Sciences and Technologies for Transport, Development and Networks, in the Transportation Engineering and Computer Science Lab GRETTIA. In 2009, he was visiting researcher at TU-Delft (Netherlands). He is since 2014 head of the "Modeling \& Multimodality" research team.

Mahdi Zargayouna is mainly interested in multiagent systems (languages, coordination models, simulation, planning, etc.), and complex transportation applications (traveler information, crisis management, dial a ride, urban parking, etc.). He teaches multiagent systems and 
intelligent transportation systems at University of Paris Dauphine, University Paris Est, EPITA Engineering School and ESIEE-Paris Engineering school. He has published more than 50 papers in peer-reviewed journals and conference proceedings and is member of the reviewer board of several international journals and conferences.

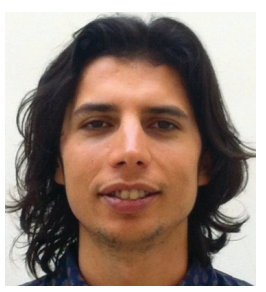

Amine Othman received his M.Sc degree in computer science from the University of Paris Dauphine (France) in 2011 and his Ph.D. in computer science and transportation science from university of Paris Est in 2016. He works with IFSTTAR, the French Institute of Sciences and Technologies for Transport, Development and Networks, in the Transportation Engineering and Computer Science Lab GRETTIA. Amine Othman is mainly interested in multiagent simulation and traveler information.

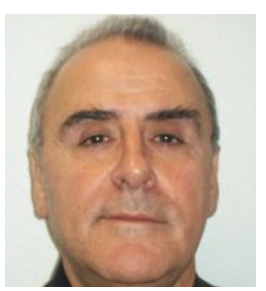

Gérard Scemama received his M.Sc degree and the Ph.D. degree in mathematics and operational research from the University of Pierre et Marie Curie (France), in 1976 and 1977 respectively. He joined IFSTTAR, the French Institute of Sciences and Technologies for Transport, Development and Networks, in 1981. He is research director since 1992 and has been director of the Transportation Engineering and Computer Science Lab GRETTIA for 14 years (from 1997 to 2011).

Gérard Scemama is mainly interested in supervision and regulation systems in multimodal networks. He is the inventor of Claire and ClaireSiti supervision systems, for road and multimodal networks respectively. During his 35 years of research, Gérard Scemama has managed several National and European projects and participated in scientific commissions of several institutes in France and Europe.

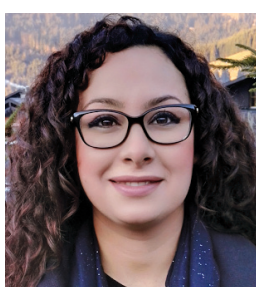

Besma Zeddini received her M.Sc degree in computer science and the Ph.D. degree in computer science and artificial intelligence from the University of Le Havre (France), in 2006 and 2009 respectively. She has been assistant professor at university of Evry and ESIGELEC engineering school for 3 years, then researcher at IFSTTAR for one year. Since 2013, she is assistant professor in computer science at EISTI engineering school, and researcher at QUARTZ lab. She is director of the "Smart Systems" option.

Besma Zeddini is mainly interested in multiagent systems, transportation systems and internet of things.
She has published more than 30 papers in peer-reviewed journals and conference proceedings and is member of the reviewer board of several international conferences.

\section{References}

[1] A. Abadi, T. Rajabioun, and P. A. Ioannou, "Traffic flow prediction for road transportation networks with limited traffic data," IEEE Trans. Intell. Transp. Syst., vol. 16, no. 2, pp. 653-662, 2015.

[2] L. Adacher, G. Oliva, and F. Pascucci, "Decentralized route guidance architectures with user preferences in urban transportation networks," Procedia-Soc. Behav. Sci., vol. 111, pp. 1054-1062, 2014.

[3] H. Bast and S. Storandt, "Frequency-based search for public transit," in Proc. 22nd ACM SIGSPATIAL Int. Conf. Advances Geographic Information Systems, 2014, pp. 13-22.

[4] A. L. Bazzan and F. Klügl, "A review on agent-based technology for traffic and transportation," Knowl. Eng. Rev., vol. 29, no. 3, pp. 375403, 2014.

[5] F. L. Bellifemine, G. Caire, and D. Greenwood, Developing Multi-Agent Systems with JADE. New York, NY, USA: Wiley, 2007.

[6] N. Bessghaier, M. Zargayouna, and F. Balbo, "Management of urban parking: An agent-based approach," in Artificial Intelligence: Methodology, Systems, and Applications. New York, NY, USA: Springer, 2012, pp. 276-285.

[7] Q. Bragard, A. Ventresque, and L. Murphy, "Self-balancing decentralized distributed platform for urban traffic simulation," IEEE Trans. Intell. Transp. Syst., pp. 1-8, 2016.

[8] C. Brakewood, S. Barbeau, and K. Watkins, "An experiment evaluating the impacts of real-time transit information on bus riders in Tampa, Florida," Transp. Res. A, vol. 69, pp. 409-422, 2014.

[9] O. Cats, H. Koutsopoulos, W. Burghout, and T. Toledo, "Effect of real-time transit information on dynamic path choice of passengers," Transp. Res. Rec: J. Transp. Res. Board, vol. 2217, pp. 46-54, 2011.

[10] S. Chipeaux, F. Bouquet, C. Lang, and N. Marilleau, "Modelling of complex systems with AML as realized in MIRO project," in Proc. IEEE Web Intelligence and Intelligent Agent Technology, 2011, pp. 159-162.

[11] P. Coppola and L. Rosati, "Simulation-based evaluation of advanced public transportation information systems (aptis)," in Schedule-Based Modeling of Transportation Networks. New York, NY, USA: Springer, 2009, pp. 1-21.

[12] K. Dziekan and K. Kottenhoff, "Dynamic at-stop real-time information displays for public transport: Effects on customers," Transp. Res. A: Pol. Pract., vol. 41, no. 6, pp. 489-501, 2007.

[13] M. Estrada, R. Giesen, A. Mauttone, E. Nacelle, and L. Segura, "Experimental evaluation of real-time information services in transit systems from the perspective of users," in Proc. Conference on Advanced Systems in Public Transport, 2015.

[14] J. Ferber, Multi-Agent Systems:An introduction to Distributed Artificial Intelligence, vol 1. Reading, MA, USA: Addison-Wesley, 1999.

[15] A. Fonzone and J. Schmöcker, Effects of Transit Real-Time Information Usage Strategies. Washington, DC, USA: Transportation Research Board, 2014.

[16] M. D. Hickman and N. H. Wilson, "Passenger travel time and path choice implications of real-time transit information," Transp. Res. C: Emerg. Technol., vol. 3, no. 4, pp. 211-226, 1995.

[17] T.-Y. Hu, C.-C. Tong, T.-Y. Liao, and W.-M. Ho, "Simulation-assignment-based travel time prediction model for traffic corridors," IEEE Trans. Intell. Transp. Syst., vol. 13, no. 3, pp. 1277-1286, 2012.

[18] M. Jakob, Z. Moler, A. Komenda, Z. Yin, A. X. Jiang, M. P. Johnson, M. Pechoucek, and M. Tambe, "Agentpolis: Towards a platform for fully agent-based modeling of multi-modal transportation (demonstration)," in Proc. Int. Conf. Autonomous Agents and Multi-Agent Systems, 2012, pp. 1501-1502.

[19] M. A. S. Kamal, J-i Imura, T. Hayakawa, A. Ohata, and K. Aihara, "A vehicle-intersection coordination scheme for smooth flows of traffic without using traffic lights," IEEE Trans. Intell. Transp. Syst., vol. 16, no. 3, pp. 1136-1147, 2015.

[20] S. Koenig and M. Likhachev, "D* lite," in Proc. Association Advancement Artificial Intelligence/Innovative Applications Artificial Intelligence, 2002, pp. 476-483.

[21] F. Ksontini, M. Zargayouna, G. Scemama, and B. Leroy, "Building a realistic data environment for multiagent mobility simulation," Smart Innovation, Syst. Technol., pp. 10, 2016.

[22] S. Luke, C. Cioffi-Revilla, L. Panait, and K. Sullivan, "Mason: A new multi-agent simulation toolkit," in Proc. SwarmFest Workshop, 2004.

[23] M. Maciejewski and K. Nagel, "Towards multi-agent simulation of the dynamic vehicle routing problem in matsim," in Proc. 9th Int. Conf. Parallel Processing and Applied Mathematics Volume Part II, Berlin, Heidelberg, 2012, pp. 551-560. 
[24] M. Mastio, M. Zargayouna, and O. Rana, "Towards a distributed multiagent travel simulation," Smart Innovation Syst. Technol., vol. 38, pp. 15-25, 2015.

[25] G. Mcardle, E. Furey, A. Lawlor, and A. Pozdnoukhov, "Using digital footprints for a city-scale traffic simulation,” ACM Trans. Intell. Syst. Technol., vol. 5, no. 3, pp. 41:1-41:16, July 2014.

[26] K. Nagel and M. Rickert, "Parallel implementation of the transims micro-simulation," Parallel Comput., vol. 27, no. 12, pp. 1611-1639, 2001.

[27] A. Othman, M. Zargayouna, B. Zeddini, and G. Scemama. "Simulating the impact of future internet on multimodal mobility," in Proc. 11th ACS/IEEE Int. Conf. Computer Systems and Applications, 2014, p. 10,

[28] F. C. Pereira, F. Rodrigues, E. Polisciuc, and M. Ben-Akiva, "Why so many people? explaining nonhabitual transport overcrowding with internet data," IEEE Trans. Intell. Transp. Syst., vol. 16, no. 3, pp. 1370-1379, 2015.

[29] K. Ramamohanarao, H. Xie, L. Kulik, S. Karunasekera, E. Tanin, R. Zhang, and E. B. Khunayn, "Smarts: Scalable microscopic adaptive road traffic simulator," ACM Trans. Intell. Syst. Technol., vol. 8, no. 2, pp. 26:1-26:22, Dec. 2016.

[30] C. L. Schweiger, Customer and Media Reactions to Real-Time Bus Arrival Information Systems. Transportation Research Board, p. 48, 2003.

[31] P. Taillandier, D.-A. Vo, E. Amouroux, and A. Drogoul, "Gama: A simulation platform that integrates geographical information data, agent-based modeling and multi-scale control," in PRIMA, volume 7057 Lecture Notes Computer Science. New York, NY, USA: Springer, pp. 242-258, 2012.

[32] E. Tatara and J. Ozik. "How to build an agent-based model III: Repast simphony," in Proc. Applied Agent-based Modeling Management Research Academy Management Annu. Meeting, Chicago, 2009.
[33] Tisseo, "press release," Feb. 24, 2011. [Online]. Accessed on: Sept. 26, 2017.

[34] L. Vercouter, "A distributed approach to design open multi-agent systems," in Engineering Societies in the Agents World II, Second International Workshop, ESAW 2001, Prague, Czech Republic, July 7, 2001, Revised Papers, volume 2203 of Lecture Notes in Computer Science, A. Omicini, P. Petta, and R. Tolksdorf, Eds. New York, NY, USA: Springer, 2001, pp. 25-38.

[35] H. C. Wong and K. Sycara, "A taxonomy of middle-agents for the internet," in Proc. 4th Int. Conf. MultiAgent Systems, 2000, pp. 465-466.

[36] M. Wooldridge and N. R. Jennings, "Intelligent agents: Theory and practice," Knowl. Eng. Rev., vol. 10, no. 2, pp. 115-152, 1995.

[37] M. Zargayouna, F. Balbo, and G. Scemama, "A multi-agent approach for the dynamic VRPTW," in Proc. Int. Workshop Engineering Societies Agents World, Saint-Etienne, 2008.

[38] M. Zargayouna and B. Zeddini, "Fleet organization models for online vehicle routing problems," in Transactions on Computational Collective Intelligence VII. New York, NY, USA: Springer, 2012, pp. 82-102.

[39] Y. Zheng, L. Capra, O. Wolfson, and H. Yang, "Urban computing: Concepts, methodologies, and applications," ACM Trans. Intell. Syst. Technol., vol. 5, no. 3, pp. 38:1-38:55, Sept. 2014.

Index Terms-Simulation, Multiagent systems, Traveler information, Public transportation 